\title{
Jet emission in NGC 1052 at radio, optical, and X-ray frequencies
}

\author{
M. Kadler ${ }^{1}$, J. Kerp ${ }^{2}$, E. $\operatorname{Ros}^{1}$, H. Falcke ${ }^{1, \star}$, R. W. Pogge ${ }^{3}$, and J. A. Zensus ${ }^{1}$ \\ 1 Max-Planck-Institut für Radioastronomie Bonn, Auf dem Hügel 69, 53121 Bonn, Germany \\ 2 Radioastronomisches Institut, Universität Bonn, Auf dem Hügel 71, 53121 Bonn, Germany \\ 3 Department of Astronomy, Ohio State University, 140 West 18th Avenue, Columbus, OH 43210-1173, USA
}

Received 28 July 2003 / Accepted 19 February 2004

\begin{abstract}
We present a combined radio, optical, and X-ray study of the nearby LINER galaxy NGC 1052. Data from a short (2.3 ks) CHANDRA observation of NGC 1052 reveal the presence of various jet-related X-ray emitting regions, a bright compact core and unresolved knots in the jet structure as well as an extended emitting region inside the galaxy well aligned with the radio synchrotron jet-emission. The spectrum of the extended X-ray emission can best be fitted with a thermal model with $k T=(0.4-0.5) \mathrm{keV}$, while the compact core exhibits a very flat spectrum, best approximated by an absorbed powerlaw with $N_{\mathrm{H}}=(0.6-0.8) \times 10^{22} \mathrm{~cm}^{-2}$. We compare the radio structure to an optical "structure map" from a Hubble Space Telescope (HST) observation and find a good positional correlation between the radio jet and the optical emission cone. Bright, compact knots in the jet structure are visible in all three frequency bands whose spectrum is inconsistent with synchrotron emission.
\end{abstract}

Key words. galaxies: individual: NGC 1052 - galaxies: individual: B 0238-084 - galaxies: active - galaxies: jets

\section{Introduction}

NGC 1052 is a nearby ${ }^{1}$ elliptical galaxy which harbors a lowluminosity active galactic nucleus (LLAGN) in its very center $\left(L_{1-100 \mathrm{GHz}}=4.4 \times 10^{40} \mathrm{erg} \mathrm{s}^{-1}\right.$; Wrobel 1984). It hosts a twosided radio jet emanating from the nucleus and reaching out to kiloparsec-scales which is, however, still fully enclosed within the stellar body of the optical galaxy. In the optical, the spectrum of NGC 1052 is characterized by strong forbidden lines from low-ionization states which has made NGC 1052 the prototypical LINER (low-ionization nuclear emission line region; Heckman 1980) galaxy. As for LINERs in general, it has long been argued whether these low-ionization lines in NGC 1052 are excited by a central photo-ionizing source (e.g., Gabel et al. 2000) or if shock heating is the dominant mechanism (e.g., Sugai \& Malkan 2000). While there is overwhelming evidence for the presence of an active galactic nucleus (AGN) in NGC 1052, the role of shocks in this galaxy is still unclear. The improved angular resolution in X-rays offered by CHANDRA makes it possible for the first time to image the distribution of

Send offprint requests to: $\mathrm{M}$. Kadler,

e-mail: mkadler@mpifr-bonn.mpg.de

* Present Adress: Radio Observatory, ASTRON, PO Box 2, 7990 AA Dwingeloo, The Netherlands.

${ }^{1} D=22.6 \mathrm{Mpc}$ (assuming $z=0.0049$, Knapp et al. 1978, and $\left.H_{0}=65 \mathrm{~km} \mathrm{~s}^{-1} \mathrm{Mpc}^{-1}\right)$. At this distance 1 arcsec corresponds to $\sim 110 \mathrm{pc}$.
X-ray emission on the same scales as accomplished by connected radio interferometers, e.g., MERLIN (Multi-Element Radio-Linked Interferometer Network). Disentangling the contributions of compact (i.e., $<1$ arcsec) nuclear and extended (i.e., $>1$ arcsec) X-ray emitting regions to the total amount of X-ray emission of NGC 1052 can serve as an important tool to study the interaction between the radio jet plasma and the ambient interstellar medium.

NGC 1052 has been observed by all major X-ray missions of the pre-CHANDRA era, like Einstein (Mc Dowell 1994), ASCA and ROSAT (Weaver et al. 1999), and Beppo Sax (Guainazzi \& Antonelli 1999). For these X-ray missions, NGC 1052 appeared as a point-like X-ray source. The X-ray spectrum of NGC 1052 is extremely flat, a finding that led to the proposal of an advection dominated accretion flow (ADAF) as the origin of the observed X-ray emission (Guainazzi et al. 2000). To model the AGN X-ray spectrum above $E \simeq$ $2 \mathrm{keV}$ absorbing column densities in excess of $10^{23} \mathrm{~cm}^{-2}$ have been discussed, supporting the idea of a high density obscuring torus, comparable to column densities found in other AGN (e.g., Malizia et al. 1997; Risaliti et al. 2002). Independent evidence for the existence of an obscuring torus at the center of NGC 1052 is obtained from Very Long Baseline Interferometry (VLBI) observations in the radio regime: on parsec-scales NGC 1052 exhibits a twin jet structure with a prominent emission gap between both jets (see e.g., Kadler et al. 2003b). The inner part of the western jet shows a 
strongly inverted radio spectrum, which was first discovered by Kellermann et al. (1999) (see also Kameno et al. 2001). The cm-wavelength spectral index in this central region is larger than 2.5, exceeding the theoretical limit for synchrotron self-absorption.

Combined studies of the core region of NGC 1052 in the radio and $\mathrm{X}$-ray regime are of essential importance for constraining the physical properties of the parsec-scale radio jet and the obscuring torus as well as to determine the nature of the nuclear X-ray emission. In this paper we present a combined radio, optical, and X-ray study of the jet-related emission in NGC 1052 on arcsecond scales. In particular, we focus on the soft X-ray excess in the source-spectrum below $E=2 \mathrm{keV}$. This soft component was identified first by Weaver et al. (1999) based on ROSAT PSPC data. CHANDRAs superior angular resolution makes it possible to present evidence that this soft excess emission is associated with the well known radio jet.

In Sect. 2 we present the CHANDRA, MERLIN, and $H S T$ data as well as their reduction. In Sect. 3 we discuss the arcsecond-scale morphology of NGC 1052 in the radio, optical, and X-ray regime and the correlations between the different wave bands. In Sect. 4 we derive models for the nuclear and extended X-ray emission and Sect. 5 summarizes our conclusions.

\section{Observations and data reduction}

\subsection{CHANDRA data}

CHANDRA observed NGC 1052 on August 29/30, $2000^{2}$. During the $2342 \mathrm{~s}$ observation, the Advanced CCD Imaging Spectrometer (ACIS) Chip S3 was in the focus of the High Resolution Mirror Assembly (HRMA). The ACIS-S3 detector offers high angular resolution as well as information on the X-ray source spectrum because of its intrinsic energy resolution and has a higher sensitivity in the soft X-ray energy regime below $E<1 \mathrm{keV}$ then the front-side illuminated detectors of CHANDRA.

The nucleus of NGC 1052 is the brightest X-ray source within the field of interest. The count rate of $0.12 \mathrm{cts} \mathrm{s}^{-1}$ is sufficiently high to affect the measured AGN X-ray spectrum by the pile-up effect. The pile-up effect changes the shape of the measured X-ray spectrum as well as the measured count rate, because during a single read-out period of the chip multiple $\mathrm{X}$-ray photons may be detected within a single pixel. Due to insufficient time resolution their combined signal is registered as a single photon event. The presented CHANDRA observation was performed using the standard timed exposure mode. Depending on the detailed shape of the AGN X-ray spectrum up to $27 \%$ of the available data can be affected by the pile-up effect. In principle, this introduces a bias which results in spectral hardening and mimics lower count rates.

The spatial distribution of photon events on the ACIS-S3 chip during the observation of NGC 1052 is shown in Fig. 1 for

\footnotetext{
2 The CHANDRA data were taken from the public archive (http://cxc.harvard.edu/cda/chaser.html) and analyzed using standard methods within the software package CIAO 3.0 using the calibration data base CALDB 2.2 .5 version.
}

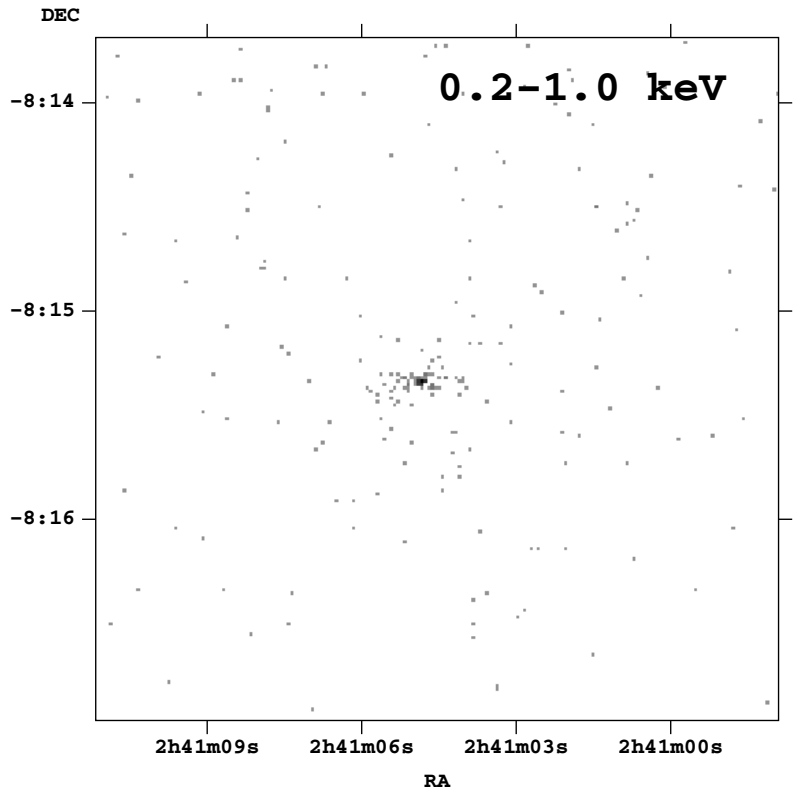

Fig. 1. Raw CHANDRA image of NGC 1052 showing the spatial distribution of photon events in the energy range $(0.2-1.0) \mathrm{keV}$.

the energy range $(0.2-1) \mathrm{keV}$. Even from the small number of counts during this snapshot observation the distribution of soft $\mathrm{X}$-rays clearly differs from a point spread function. Using the CSMOOTH program which is part of the CIAO software we also produced an adaptively smoothed map of the field of interest (see Fig. 2) for which we reduced the angular resolution of the data from 0.5 arcsec to 4 arcsec and set a minimum significance threshold to $3 \sigma$.

\subsection{MERLIN data}

A MERLIN observation of NGC 1052 at $1.4 \mathrm{GHz}$ was performed on November 22, 1995. The data from this experiment have been obtained from the public archive ${ }^{3}$ and analyzed applying standard methods using the program DIFMAP. Two different maps of the brightness distribution of NGC 1052 at $1.4 \mathrm{GHz}$ were produced. First, a strong $(u, v)$-taper was used to map the extended emission resulting in a restoring beam of $(1.5 \times 1.1)$ arcsec at a position angle (PA) of $-31^{\circ}$. Second, a pure naturally weighted image was produced yielding a restoring beam of $(0.5 \times 0.3) \operatorname{arcsec}$ at a PA of $27^{\circ}$.

\subsection{HST data}

For this paper, we re-examined the archival HST data of NGC 1052 previously published by Pogge et al. (2000). The archival data are three narrow-band $F 658 N$ filter images acquired with the WFPC2 in the PC1 detector (pixels scale $\sim 0.046$ arcsec) with integration times of 200, 800, and $900 \mathrm{~s}$, respectively. Cosmic rays were cleaned from the individual images using a version of the L.A.Cosmic Laplacian edge-detection algorithm described by van Dokkum (2001). The cleaned images were added together after registration

\footnotetext{
${ }^{3}$ http://www.merlin.ac.uk
} 


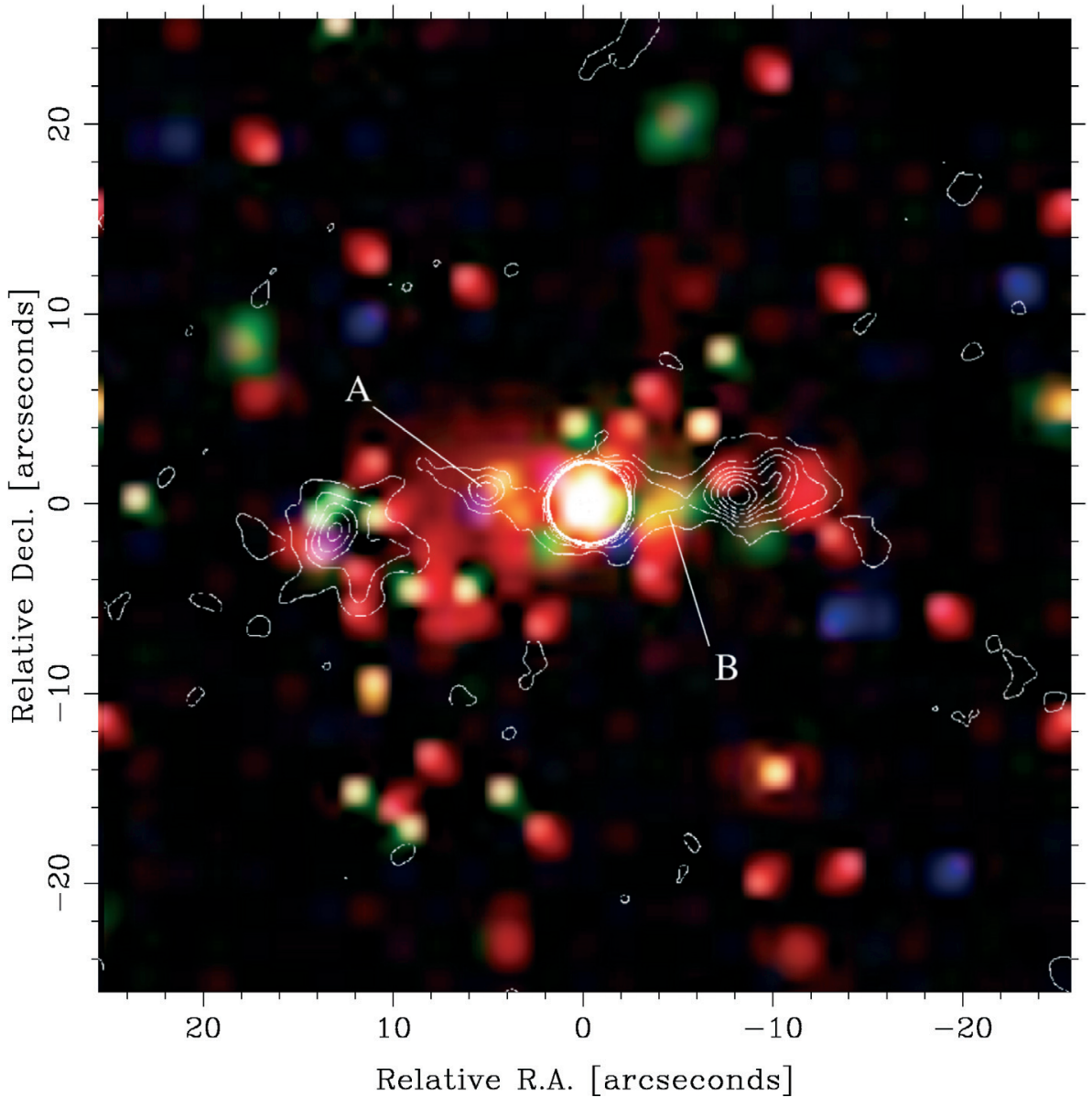

Fig. 2. Three color CHANDRA image of the jet-associated X-ray emission in NGC 1052 with the $1.4 \mathrm{GHz}$ MERLIN radio image overlaid in contours. The CHANDRA image has been smoothed to a resolution of $4^{\prime \prime}$ to increase sensitivity. Red represents photons between $0.1 \mathrm{keV}$ and $1 \mathrm{keV}$, green between $1 \mathrm{keV}$ and $2 \mathrm{keV}$ and blue (2-3) keV. The clipping level is $3 \sigma$. The MERLIN image was restored with a circular beam of 1.7 arcsec FWHM. Contours are shown for $(0.5,1.5,2.5,3.5,4.5,5.5$, $6.5) \times 1 \mathrm{mJy} /$ beam. The jet-associated emission is dominated by soft $\mathrm{X}$-rays below $1 \mathrm{keV}$, whereas the AGN appears white, representing a rather flat $\mathrm{X}$-ray spectrum. using a field star in the image to remove the inter-image offsets. To enhance the faint emission features noted by Pogge et al. (2000), we created a "structure map" of the combined, cleaned images following the technique described by Pogge $\&$ Martini (2002). We used a model point spread function for the $F 658 N$ filter and PC1 camera generated using the TINYTIM package (Krist \& Hook 1999). Structure mapping is an improved method of image contrast enhancement (compared to traditional unsharp masking). In brief, the technique suppresses the large-scale starlight distribution, enhancing faint emission and absorption (e.g., dust extinction) features in the image.

\section{The multi-waveband jet structure}

\subsection{Jet-associated X-ray emission in NGC 1052}

The CHANDRA image of NGC 1052 in the energy range $(0.3-3.0) \mathrm{keV}$ is shown in Fig. 2. Strong X-ray emission from the AGN is seen in this image, as well as diffuse extended emission well aligned with the radio jet, whose MERLIN image is superimposed in contours. In Sects. 4.1 and 4.2 we discuss the $\mathrm{X}$-ray spectra in detail and present evidence that the soft excess component found previously in the ROSAT spectra of the nucleus (Weaver et al. 1999) is associated with this soft, extended jet-associated emission.

To the first order, the radio and the X-ray jet are aligned on arcsecond scales and their extent appears to be the same.
However, the intensity of synchrotron emission in the radio lobes appears to be anti-correlated with the soft X-ray intensity distribution in so far as the radio hotspots on both sides correspond to regions on the ACIS-S3 chip which did not detect any photon events. East and west of the nucleus two relatively bright X-ray emitting regions coincide with emission knots in the radio regime. Bright optical emission knots are also present in these areas (Pogge et al. 2000).

\subsection{Radio emission on arcsecond scales}

The large-scale radio structure of NGC 1052 visible in the tapered MERLIN image reveals some differences to the 1980 VLA image of Wrobel (1984) (see Fig. 3). The VLA, which was operated in its A configuration during the $1980 \mathrm{ob}-$ servations, was more sensitive to extended structures, which partially have been resolved out by MERLIN. The core has varied, with a flux density of $\sim 0.74 \mathrm{Jy}$ in December 1980 and $\sim 1.01 \mathrm{Jy}$ in November 1995 . The western hot spot has increased in flux density. A knot in the western jet (labeled as $\mathrm{B}$ in the MERLIN image) was not visible as a local maximum in the 1980 VLA image. By aligning the core positions at both epochs, we found that the eastern knot (labeled as A), has moved about 0.5 arcsec inwards. However, an examination of the pure naturally weighted MERLIN image (see Fig. 4) reveals that knot $\mathrm{A}$ is composed of two isolated sub-components (A 1 and A 2) separated by the same distance of $\sim 0.5$ arcsec. 


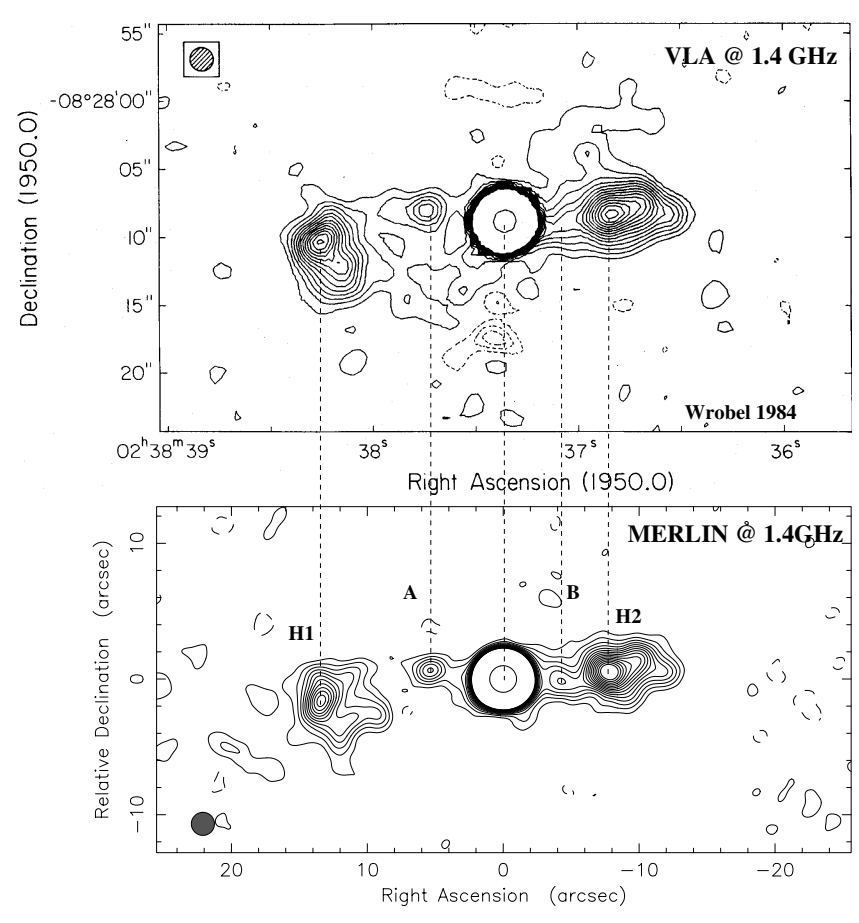

Fig. 3. Comparison between the VLA image of NGC 1052 observed in 1980 (Wrobel 1984) and the MERLIN image from 1995, both at $1.4 \mathrm{GHz}$. Both maps have been convolved with the same (circular) restoring beam of 1.7 arcsec $F W H M$. Contours at $(-1,1,2,3,4$, $5,6,7,8,9,10,11,12,13,14,15,800) \times 0.5 \mathrm{mJy}$ are given in both maps (the VLA map also shows negative contours at $(-4,-3,-2) \times$ $0.5 \mathrm{mJy}$ ). The hot spots (labeled as $\mathrm{H} 1$ and $\mathrm{H} 2$ ) do not show any positional changes. The emission peak of the eastern knot (A) is about 0.5 arcsec closer to the core in 1995 .

This suggests that the inner sub-component might have increased in flux density between 1980 and 1995, causing an apparent shift of the blended knot structure in Fig. 2. This offers a more plausible explanation than an inward motion with a velocity of $\sim 10 c$.

\subsection{Optical structure}

The structure map of NGC 1052 is shown in Fig. 4 with the radio contours of the untapered, full-resolution MERLIN image superimposed. The jet-counterjet structure is aligned with the optical emission cone. The dark band perpendicular to the radio jet might be an artifact of the image processing. Two optical emission knots are located at the edges of the two radio subcomponents of knot A. The optical emission knot in the west coincides roughly with a weak $(\sim 1 \sigma)$ radio feature while the stronger radio knot 1.5 arcsec further out has no corresponding bright optical counterpart. The origin of the optical emission remains unclear since there is no continuum image to subtract from the $\mathrm{H} \alpha$ filter image of NGC 1052. The conical morphology in this LINER 1.9 galaxy is remarkably similar to the structure of the narrow-emission-line region typically observed in Seyfert 2 galaxies (e.g., Falcke et al. 1998). This suggests that the optical emission cone is due to line emission rather than continuum emission. The optical flux density of the two eastern knots of $(68 \pm 1) \mu \mathrm{Jy}$ exceeds the power-law extrapolation from the radio to the X-ray regime by almost three orders of magnitude. This is a strong argument against the synchrotron emission process, but compatible with relatively strong line emission exceeding the contimuum emission of the knots in this narrow band.

\section{X-ray imaging spectroscopy}

Using CHANDRA's high angular resolution, it is possible to obtain separate spectra of the nucleus and the diffuse jetassociated X-ray emission. We selected an annulus centered at the nucleus position which excludes the nucleus itself but includes the whole area of the diffuse emission. To avoid any contamination by the nuclear X-ray emission, a diameter of 3 arcsec for the inner annulus ring was used. The nuclear $\mathrm{X}$-ray spectrum was extracted up to a diameter of 2 arcsec. The extracted X-ray data of both regions were corrected for the unrelated X-ray background emission, using a region located close by without significant point sources. We also applied a correction for the quantum efficience degeneracy of the ACIS detector.

Figure 5 reveals the much softer spectrum of the diffuse $\mathrm{X}$-ray emission compared to the nuclear spectrum. The bulk emission originates below $E<2 \mathrm{keV}$, while the X-ray spectrum of the nucleus has an additional hard X-ray component. Remarkable is the soft X-ray emission below $E<2 \mathrm{keV}$ towards the nucleus. ASCA, as well as the ROSAT PSPC, could not separate the nucleus and the jet spatially, but a soft excess X-ray emission had already been detected in the X-ray spectrum from the PSPC data.

\subsection{The $X$-ray spectrum of the extended emission}

To constrain the emission process of the diffuse extended emission, we fitted different models using XSPEC (version 11.2): the RAYMOND model corresponding to a Raymond/Smith plasma (Raymond \& Smith 1977), the MEKAL model describing a Mewe/Kaastra plasma (e.g., Kaastra 1992) and a simple powerlaw. The results of the spectral fits are presented in Table 1.

Because the jet is located deep inside the stellar body of the galaxy NGC 1052, we have to determine the amount of photoelectric absorption distributed along the line of sight. Using the thermal source models, we find an attenuating column density value about one order of magnitude higher than the galactic foreground column density belonging to the Milky Way (Hartmann \& Burton 1997). We attribute this additional X-ray attenuation to weakly ionized gas located inside the galaxy itself. The unabsorbed flux of the Raymond/Smith (Mewe/Kaastra) plasma in the range between 0.3 and $8 \mathrm{keV}$ corresponds to an intrinsic luminosity of $L=2.4(2.1) \times$ $10^{40} \mathrm{erg} \mathrm{s}^{-1}$ at the distance of the source of $22.6 \mathrm{Mpc}$.

Both plasma models fall below the measured count rate above $2.5 \mathrm{keV}$ (see Fig. 5) suggesting some contribution of an additional hard X-ray component to the spectrum of the extended emission. Due to the low photon statistics and the large uncertainties we have not tried to account for this additional spectral component by considering more complicated models. 


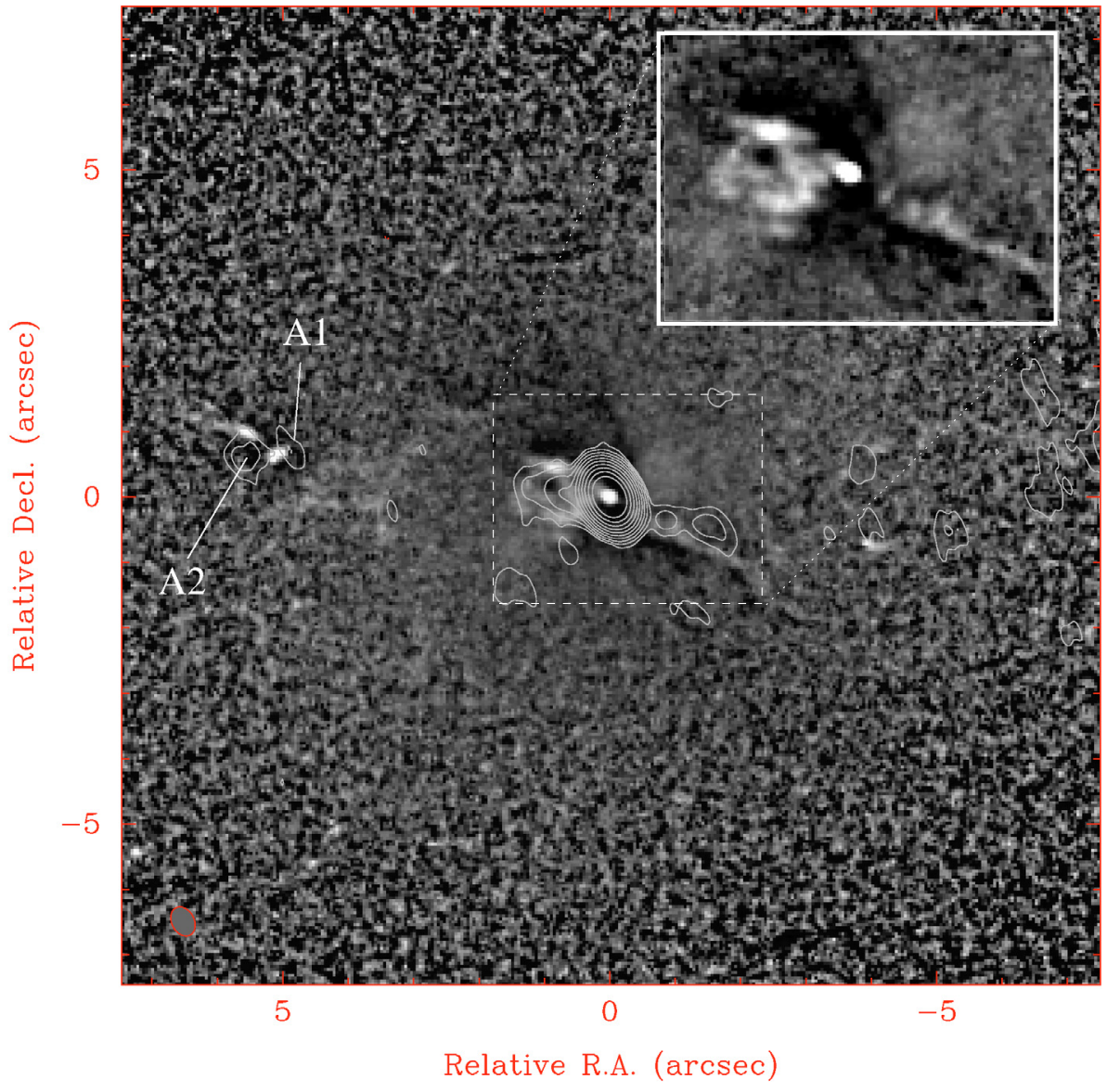

Fig. 4. HST structure map of NGC 1052. Dark regions represent dust obscuration while bright regions are locations of enhanced emission. Overlaid is the pure naturally weighted MERLIN map showing the core of NGC 1052 with sub-arcsecond resolution. Contours at $(-1,1,2,4,8,16,32$, $64,128,256,512,1024) \times 0.5 \mathrm{mJy} /$ beam are shown. The inlaid panel shows an enlarged view of the marked box around the area of the optical emission cone.
Because of the low number of counts from the jet, we cannot unequivocally dismiss the power-law model, which gave an acceptable fit to the X-ray data. However, we consider the power-law fit result of the X-ray jet as unreliable because of the unusually steep photon index ${ }^{4}$ (see Table 1 ).

\subsection{The $X$-ray spectrum of the nucleus}

Below $E=2 \mathrm{keV}$ a soft excess is detected in the nuclear spectrum. Assuming that a fraction of the diffuse jet-associated emission originates within the immediate neighborhood of the nucleus, we used a hybrid model for the X-ray spectral approximation of the observed intensity distribution.

$I_{\text {obs }}=\left(I_{\text {diffuse }}+I_{\text {nucleus }} \times \mathrm{e}^{-\sigma \times N_{\mathrm{H}(\text { torus })}}\right) \times \mathrm{e}^{-\sigma \times N_{\mathrm{H}(\text { gal. })}}$

where $I_{\text {diffuse }}$ is the X-ray spectrum of the diffuse jet-associated emission and $I_{\text {nucleus }}$ is the spectrum of the nucleus. Here, the photoelectric absorption produced by the torus is represented by $\mathrm{e}^{-\sigma \times N_{\mathrm{H}(\text { torus })}}$, while the X-ray absorption produced by the interstellar medium of the elliptical galaxy is $\mathrm{e}^{-\sigma \times N_{\mathrm{H} \text { (gal. }} \text {. }}$.

We assumed a power-law type X-ray spectrum of the central X-ray source and modeled the soft component as a thermal plasma spectrum of the Raymond/Smith and Mewe/Kaastra type, (model I and II) respectively. We did not try to fit for

\footnotetext{
${ }^{4}$ The photon index $\Gamma$ is defined as the power-law index of the spectrum given in units of [photons $\mathrm{s}^{-1} \mathrm{keV}^{-1}$ ]. It is related to the energy index $\alpha$, used in radio astronomy, via: $\Gamma=1-\alpha$.
}

the metallicities and fixed these during the spectral fitting procedure given the poor statistics from the short integration time. Model Ia(IIa) assumes solar abundances of the Raymond/Smith (Mewe/Kaastra) plasma and allows the other parameters to be varied. In model $\mathrm{Ib}(\mathrm{IIb})$ the temperatures of the plasma and the absorbing column density of the interstellar medium of the galaxy were fixed to the best fitting values to the extended X-ray emission (see Table 1). In model Ic(IIc) the metal abundances were additionally changed to $25 \%$ of the solar composition. Such low metal abundances typically occur only in dwarf galaxies but are found in NGC 1052 from optical line measurements of Sil'chenko (1995) who reports values $[\mathrm{Fe} / \mathrm{H}] \sim-0.6$ for the nucleus and $[\mathrm{Fe} / \mathrm{H}] \sim-1$ for the bulge of NGC 1052, corresponding to metallicities of about $25 \%$ and $10 \%$, respectively. One way to accumulate a substantial amount of metalpoor material is a merger or close encounter with an extremely metal-poor dwarf galaxy. Evidence for such an event indeed exists from multiple observational approaches (see Forbes et al. 2001 and references therein) in the case of NGC 1052.

The statistically best fitting values are given in Table 2 . For all six models we derive a luminosity of $L=1.4 \times 10^{41} \mathrm{erg} \mathrm{s}^{-1}$. Correcting for the effect of the two absorber model components increases the intrinsic (unabsorbed) value to $L=(1.7-2.0) \times$ $10^{41} \mathrm{erg} \mathrm{s}^{-1}$. The values of $N_{\mathrm{H}(\mathrm{gal} .)}$ and $k T$ for the plasma component in the models Ia and IIa are very similar to the values determined for the extended plasma emission. For these models we derive best fitting values for the absorbing column density of $N_{\mathrm{H} \text { (torus) }}=(0.6-0.7) \times 10^{22} \mathrm{~cm}^{-2}$ as well as $\Gamma=0.2-0.3$ 

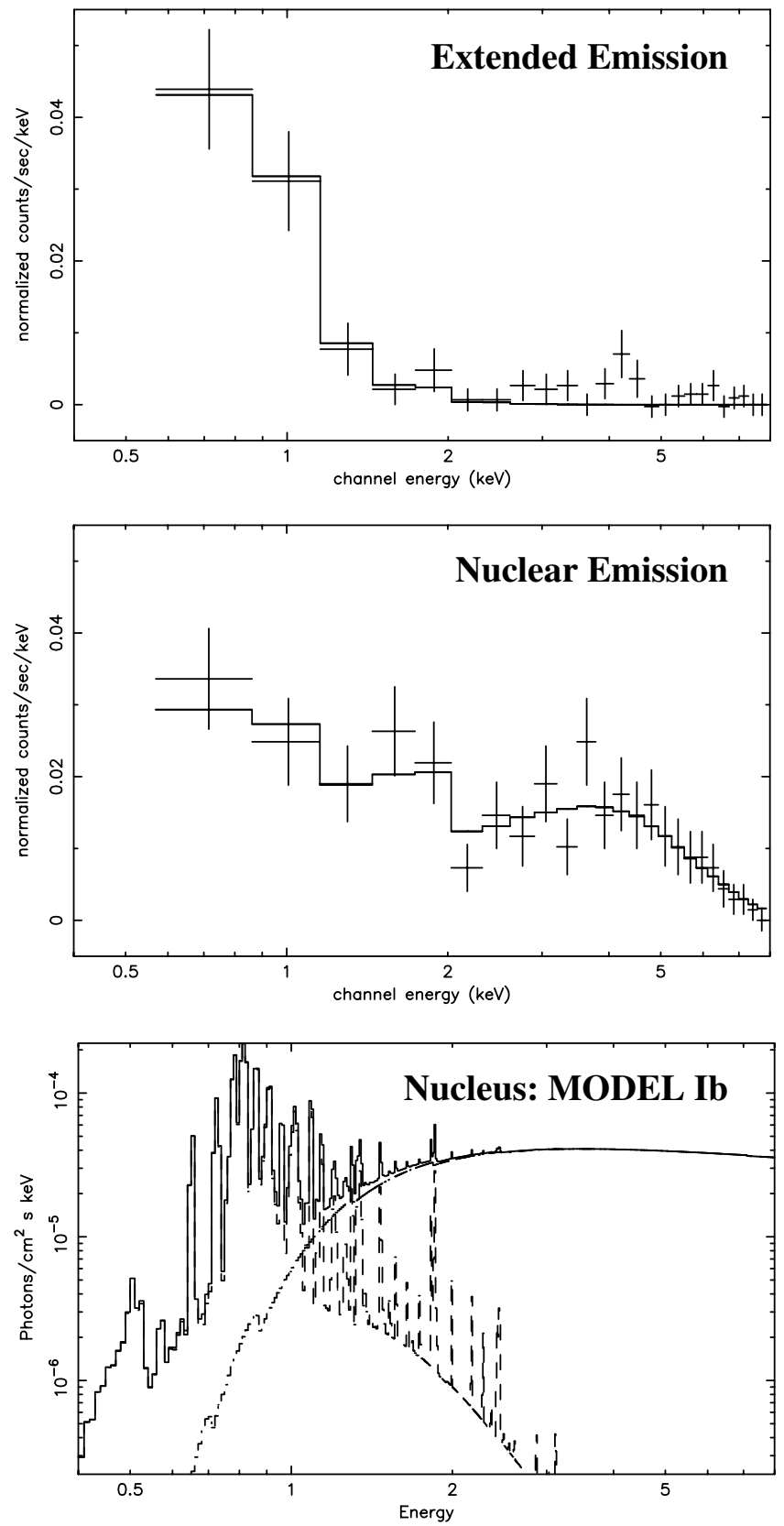

Fig. 5. Top panel: X-ray spectrum of the extended X-ray jet emission and the Raymond/Smith plasma model folded with the detector response matrix (solid line). Middle panel: X-ray spectrum of the nuclear emission and the best fit of model Ib (solid line, compare Table 4.2). Bottom panel: the unfolded model Ib spectrum representing an absorbed Raymond/Smith plasma whose model parameters have been held fixed at the best fitting values to the extended X-ray emission spectrum (see Table 1) plus a power-law with an additional absorber.

for the photon index. Similar values for both parameters result from fixing $N_{\mathrm{H} \text { (gal.) }}$ and $k T$ as described above in the models Ib and IIb. Reducing the metal abundances of the plasma component in model Ic(IIc) forces the power-law to contribute more strongly to the soft part of the spectrum resulting in higher values of $N_{\mathrm{H} \text { (torus) }} \sim 0.8 \times 10^{22} \mathrm{~cm}^{-2}$ and $\Gamma \sim 0.3$. These values are still rather low. Data from other X-ray observatories imply much higher values for $N_{\mathrm{H} \text { (torus) }}$ as well as for $\Gamma$ (Weaver et al. 1999; Guainazzi \& Antonelli 1999; Guainazzi et al. 2000). We note that the apparent discrepancy between previous X-ray observations and the CHANDRA data concerning the photon index might be due to the pile-up degradation $(\sim 30 \%)$ of the latter. The nuclear X-ray spectrum thus might appear artificially flattened. The determined absorbing column density, however, is not so sensitive to the pile-up effect, which mainly affects the hard part of the spectrum while the amount of absorption is determined in the soft X-ray regime. The statistical quality of all six fits is very similar, with values of $\chi_{\text {red }}^{2}$ ranging from 0.76 to 0.84 , and the low photon statistics (reflected in the values of $\chi_{\text {red }}^{2}<1$ ) do not allow us to dismiss any of these models.

We note that a "patchy" absorber model in which a fraction of the central source is seen directly and only a part of the source is covered by the absorber might be a more realistic model for the nuclear X-ray emission of NGC 1052, given the results of Vermeulen et al. (2003), who found that moving VLBI components in both jets show complex light curves probably caused by substantial patchiness of the absorbing screen. Additionally, they find a complex H I absorption line spectrum with compact clouds of absorbing material at different relative velocities and different locations along the parsec-scale jet structure. The absorbing column density derived from "patchy" absorber models is expected to be considerably higher $\left(\sim 2 \times 10^{22} \mathrm{~cm}^{-2}\right)$ than the values derived from uniform absorber models. However, given the low photon statistics, we omit the detailed discussion of such models with a larger number of free parameters.

\section{Discussion}

The CHANDRA data provide for the first time direct evidence for jet-associated X-ray emission in NGC 1052. The diffuse, extended X-ray emission can be best approximated with a thermal plasma model with $k T \sim 0.4-0.5 \mathrm{keV}$. This temperature is consistent with the thermal component found earlier by Weaver et al. (1999) using ASCA and ROSAT data. Its absorbed flux is only $\sim 3 \%$ of the nuclear X-ray emission but the intrinsic (absorption corrected) extended emission might contribute up to $14 \%$ to the total unabsorbed X-ray flux of NGC 1052. Because of the considerable pile-up degradation of the CHANDRA data, no firm conclusions on the photon index of the nucleus spectrum can be deduced. The derived column density of hydrogen towards the compact X-ray core (depending on the applied model) of $0.5-0.8 \times 10^{22} \mathrm{~cm}^{-2}$ is in good agreement with the absorbing column density of ionized material towards the VLBI-jet derived by Kadler et al. (2002) and Kadler et al. (in prep.). This suggests that the nuclear X-ray emission of NGC 1052 might be produced on the same scales as the parsec-scale structures imaged by VLBI at high frequencies.

The detection of a diffuse region of X-ray emitting gas with a thermal spectrum and the same extent as the kiloparsec-scale radio jet suggests that jet-triggered shocks might play an important role in NGC 1052. In such a model the kinetic power of the radio jet is partially converted into X-ray emission. The optical morphology in the $\mathrm{H} \alpha$ filter substantiates this picture as was 
Table 1. Best-fitting parameters for the spectral fits to the extended jet-associated emission.

\begin{tabular}{ccccccc}
\hline \hline Model $^{(a)}$ & $\begin{array}{c}N_{\mathrm{H}}^{(b)} \\
{\left[10^{22} \mathrm{~cm}^{-2}\right]}\end{array}$ & $\begin{array}{c}k T \\
{[\mathrm{keV}]}\end{array}$ & $\Gamma^{(c)}$ & $\begin{array}{c}F_{\text {abs }}^{(d)} \\
{\left[\mathrm{erg} \mathrm{s}^{-1} \mathrm{~cm}^{-2}\right]}\end{array}$ & $\begin{array}{c}F_{\text {unabs }}^{(d)} \\
{\left[\mathrm{erg} \mathrm{s}^{-1} \mathrm{~cm}^{-2}\right]}\end{array}$ & $\chi_{\text {red }}^{2}\left(\chi^{2} /\right.$ d.o.f. $)$ \\
\hline$(\mathrm{R} \& S)^{\mathrm{abs}}$ & $0.49_{-0.06}^{+0.08}$ & $0.48_{-0.08}^{+0.08}$ & - & $0.8 \times 10^{-13}$ & $3.9 \times 10^{-13}$ & $0.49(10.8 / 22)$ \\
$(\mathrm{M} \& \mathrm{~K})^{\mathrm{abs}}$ & $0.41_{-0.06}^{+0.08}$ & $0.41_{-0.07}^{+0.09}$ & - & $0.7 \times 10^{-13}$ & $3.4 \times 10^{-13}$ & $0.53(11.6 / 22)$ \\
$(\mathrm{PL})^{\mathrm{abs}}$ & $0.06_{-0.02}^{+0.07}$ & - & $3.8_{-0.7}^{+0.6}$ & $1.5 \times 10^{-13}$ & $2.0 \times 10^{-13}$ & $0.52(11.5 / 22)$ \\
\hline
\end{tabular}

${ }^{a}$ R\&S: Raymond/Smith plasma, M\&K: Mewe/Kaastra plasma, PL: power-law; ${ }^{b}$ absorbing column density; $2 \times 10^{20} \mathrm{~cm}^{-2}$ is set as a lower limit; ${ }^{c}$ photon index; ${ }^{d}$ (un)absorbed X-ray flux between $0.2 \mathrm{keV}$ and $8.0 \mathrm{keV}$.

Table 2. Best-fitting parameters for the spectral fits of the core emission.

\begin{tabular}{|c|c|c|c|c|c|c|c|c|}
\hline Model $^{(a)}$ & $\begin{array}{c}N_{\mathrm{H}(\text { gal. })}^{(b)} \\
{\left[10^{22} \mathrm{~cm}^{-2}\right]}\end{array}$ & $\begin{array}{c}k T \\
{[\mathrm{keV}]}\end{array}$ & $Z^{(c)}$ & $\begin{array}{c}N_{\mathrm{H}(\text { torus })}^{(d)} \\
{\left[10^{22} \mathrm{~cm}^{-2}\right]}\end{array}$ & $\Gamma^{(e)}$ & $\begin{array}{c}F_{\mathrm{abs}}^{(f)} \\
{\left[\mathrm{erg} \mathrm{s}^{-1} \mathrm{~cm}^{-2}\right]}\end{array}$ & $\begin{array}{c}F_{\text {unabs }}^{(g)} \\
{\left[\mathrm{erg} \mathrm{s}^{-1} \mathrm{~cm}^{-2}\right]}\end{array}$ & $\chi_{\text {red }}^{2}\left(\chi^{2} /\right.$ d.o.f. $)$ \\
\hline Ia & $0.66_{-0.07}^{+0.07}$ & $0.44_{-0.06}^{+0.07}$ & $1^{*}$ & $0.74_{-0.39}^{+0.56}$ & $0.30_{-0.08}^{+0.10}$ & $2.3 \times 10^{-12}$ & $3.3 \times 10^{-12}$ & $0.84(15.9 / 19)$ \\
\hline $\mathrm{Ib}$ & $0.49^{*}$ & $0.48^{*}$ & $1^{*}$ & $0.54_{-0.34}^{+0.46}$ & $0.20_{-0.08}^{+0.10}$ & $2.3 \times 10^{-12}$ & $2.9 \times 10^{-12}$ & $0.76(16.0 / 21)$ \\
\hline Ic & $0.49^{*}$ & $0.48^{*}$ & $0.25^{*}$ & $0.84_{-0.39}^{+0.55}$ & $0.28_{-0.09}^{+0.09}$ & $2.3 \times 10^{-12}$ & $3.0 \times 10^{-12}$ & $0.74(15.8 / 21)$ \\
\hline IIa & $0.51_{-0.04}^{+0.09}$ & $0.28_{-0.04}^{+0.03}$ & $1^{*}$ & $0.45_{-0.21}^{+0.59}$ & $0.22_{-0.05}^{+0.13}$ & $2.5 \times 10^{-12}$ & $3.4 \times 10^{-12}$ & $0.89(16.8 / 19)$ \\
\hline $\mathrm{IIb}$ & $0.41^{*}$ & $0.41^{*}$ & $1^{*}$ & $0.62_{-0.37}^{+0.46}$ & $0.23_{-0.09}^{+0.06}$ & $2.3 \times 10^{-12}$ & $2.8 \times 10^{-12}$ & $0.77(16.1 / 21)$ \\
\hline IIc & $0.41^{*}$ & $0.41^{*}$ & $0.25^{*}$ & $0.82_{-0.42}^{+0.42}$ & $0.27_{-0.11}^{+0.07}$ & $2.2 \times 10^{-12}$ & $2.8 \times 10^{-12}$ & $0.78(16.4 / 21)$ \\
\hline
\end{tabular}

* Fixed value; ${ }^{a} \mathrm{I}:\left(\mathrm{R} \& \mathrm{~S}+\mathrm{PL}^{\mathrm{abs}}\right)^{\mathrm{abs}}$, II: $\left(\mathrm{M} \& \mathrm{~K}+\mathrm{PL}^{\mathrm{abs}}\right)^{\mathrm{abs}}$, $\left(\mathrm{R} \& \mathrm{~S}^{\mathrm{abs}}\right.$ : absorbed Raymond/Smith plasma, M\&K ${ }^{\text {abs }}$ : absorbed Mewe/Kaastra plasma, $\mathrm{PL}^{\text {(abs) }}$ : (absorbed) power-law; ${ }^{b}$ column density of the diffuse absorber; ${ }^{c}$ metallicity (referring to the solar value); ${ }^{d}$ column density of the compact absorber; ${ }^{e}$ photon index; ${ }^{f}$ absorbed X-ray flux between $0.2 \mathrm{keV}$ and $8.0 \mathrm{keV} ;{ }^{g}$ unabsorbed X-ray flux between $0.2 \mathrm{keV}$ and $8.0 \mathrm{keV}$, i.e., $N_{\mathrm{H}(\text { gal. })}^{(b)}$ and $N_{\mathrm{H}(\text { torus })}^{(d)}$ are set to 0.

noted earlier by Allen et al. (1999). The alignment of the radio jet and the optical emission cone visible in Fig. 4 implies that the ionization cone might be drilled out by the radio jets, resulting in a predominantly shock-excited, conical narrow-line region (see e.g., Dopita 2002). Shocks might occur also on larger scales giving rise to the soft thermal $\mathrm{X}$-ray emission associated with the radio jet/lobe structure in NGC 1052. A rough estimate (see Kadler et al. 2003a) shows that the soft thermal X-ray spectrum associated with the radio jet of NGC 1052 can be explained in terms of the kinetic jet power being partially converted into $\mathrm{X}$-ray emission originating in shocks driven into the ambient medium. (A more detailed model of the relation between jet-driven shock-activity and the spectral shape of the extended X-ray emission in NGC 1052 will be discussed in a forthcoming paper.) The comparison of the large-scale distribution of radio emission in NGC 1052 between two epochs separated by $\sim 15$ years indeed shows activity on kiloparsecscales. This substantiates the idea that shocks in the interstellar medium form at the working surfaces of active regions (hotspots and knots). Moreover, recent numerical simulations (e.g., Zanni et al. 2003) show that jets in radio galaxies can inflate over-pressured cocoons that drive shocks into the ambient gas resulting in morphologies (in the case of weak shocks) very similar to what is observed in NGC 1052: a cavity of hot X-ray emitting gas in conjunction with a local deficit of X-ray emission around the hotspots. A deeper CHANDRA observation with an improved photon statistic compared to the observation discussed here would provide both a higher sensitivity to the weak diffuse emission and a higher resolution. Additionally, the full resolution of CHANDRA of $\sim 0.5$ arcsec would allow one to study in more detail the connection between the knots in the diffuse X-ray emission and the optical emission knots.

Acknowledgements. We thank G. V. Bicknell for helpful discussions and important suggestions. This research used the CHANDRA Data Archive (CDA) which is part of the CHANDRA X-ray Observatory Science Center (CXC) which is operated for NASA by the Smithsonian Astrophysical Observatory. We used CHANDRA data from an experiment planned and scheduled by G. P. Garmire. We made use of the data archive at the Space Telescope Science Institute which is operated by the Association of Universities for Research in Astronomy, Inc. under NASA contract NAS 5-26555. MERLIN is a National Facility operated by the University of Manchester at Jodrell Bank Observatory on behalf of PPARC. We made use of public MERLIN data from an experiment planned and scheduled by A. Pedlar. This research has made use of NASA's Astrophysics Data System.

\section{References}

Allen, M. G., Koratkar, A. P., \& Dopita, M. A. 1999, BAAS, 31, 893 Dopita, M. A. 2002, Rev. Mex. Astron. Astrofis., 13, 177

Falcke, H., Wilson, A. S., \& Simpson, C. 1998, ApJ, 502, 199

Forbes, D. A., Georgakakis, A. E., \& Brodie, J. P. 2001, MNRAS, 325, 1431

Gabel, J. R., Bruhweiler, F. C., Crenshaw, D. M., Kraemer, S. B., \& Miskey, C. L. 2000, ApJ, 532, 883

Guainazzi, M., \& Antonelli, L. A. 1999, MNRAS, 304, L15 
Guainazzi, M., Oosterbroek, T., Antonelli, L. A., \& Matt, G. 2000, A\&A, 364, L80

Hartmann, D., \& Burton, W. B. 1997, An Atlas of galactic neutral hydrogen (Cambridge, New York: Cambridge University Press)

Heckman, T. M. 1980, A\&A, 87, 152

Kadler, M., Ros, E., Kerp, J., et al. 2002, in Proc. of the 6th European VLBI Network Symp., ed. E. Ros, R. W. Porcas, A. P. Lobanov, \& J. A. Zensus (Bonn, Germany: MPIfR), 167

Kadler, M., Ros, E., Kerp, J., et al. 2003a, New Astron. Rev., 47, 569

Kadler, M., Ros, E., Zensus, J. A., Lobanov, A. P., \& Falcke, H. 2003b, SRT: the impact of large antennas on radio astronomy and space science, ed. N. D’Amico, F. Fusi Pecci, I. Porceddu, \& G. Tofani (Bologna: Società Italiana di Fisica), 219

Kameno, S., Sawada-Satoh, S., Inoue, M., Shen, Z., \& Wajima, K. 2001, PASJ, 53, 169

Kaastra, J. S. 1992, An X-ray Spectral Code for Optically Thin Plasmas (Internal SRON-Leiden Report, updated version 2.0)

Kellermann, K. I., Vermeulen, R. C., Cohen, M. H., \& Zensus, J. A. 1999, BAAS, 31, 856

Knapp, G. R., Faber, S. M., \& Gallagher, J. S. 1978, AJ, 83, 139
Krist, J., \& Hook, R. 1999, The Tiny Tim User's Guide, Ver. 5.0 (Baltimore: STScI)

Malizia, A., Bassani, L., Stephen, J. B., Malaguti, G., \& Palumbo, G. C. C. 1997, ApJS, 113, 311

Mc Dowell, J. C. 1994, in Einstein Obs. Unscreened IPC Data Archive Pogge, R. W., Maoz, D., Ho, L. C., \& Eracleous, M. 2000, AJ, 532, 323

Pogge, R. W., \& Martini, P. 2002, ApJ, 569, 624

Raymond, J. C., \& Smith, B. W. 1977, ApJS, 35, 419

Risaliti, G., Elvis, M., \& Nicastro, F. 2002, ApJ, 571, 234

Sil'chenko, O. K. 1995, Astron. Lett., 21, 283

Sugai, H., \& Malkan, M. A. 2000, ApJ, 529, 219

van Dokkum, P. G. 2001, PASP, 113, 1420

Vermeulen, R. C., Ros, E., Kellermann, K. I., et al. 2003, A\&A, 401, 113

Weaver, K. A., Wilson, A. S., Henkel, C., \& Braatz, J. A. 1999, ApJ, 520,130

Wrobel, J. M. 1984, ApJ, 284, 531

Zanni, C., Bodo, G., Rossi, P., et al. 2003, A\&A, 402, 949 